\title{
Mucin Family Genes are Downregulated in Colorectal Cancer Patients
}

\author{
Mohammad Azhar Aziz*, Majed AlOtaibi, Abdulkareem AlAbdulrahman, Mohammed AlDrees and Ibrahim AlAbdulkarim \\ Department of Medical Genomics, KIng Abdullah Intl. Med. Res. Ctr., King Saud Bin Abdul Aziz University for Health Sciences, Riyadh, Saudi Arabia
}

\begin{abstract}
Mucins are very well known to be associated with different types of cancer. Their role in colorectal cancer has been extensively studied without direct correlation with their change in expression levels. In the present study we employed the human exon array from Affymetrix to provide evidence that mucin family genes are downregulated in colorectal cancer tumor samples. We analyzed 92 samples taken from normal and tumor tissues. All mucin family genes except MUCL1 were downregulated with the fold change value ranging from -3.53 to 1.78 as calculated using AltAnalyze software. Maximum drop in RNA transcripts were observed for MUC2 with a fold change of -3.53 . Further, we carried out Integromics analysis to analyze mucin genes using hierarchical clustering. MUC1 and MUC4 were found to be potential biomarkers for human colorectal cancer. Top upstream regulators were identified for mucin genes. Network analyses were carried out to further our understanding about potential mechanisms by which mucins can be involved in causing colorectal cancer.
\end{abstract}

Keywords: Microarray; Colorectal cancer; Mucins

\section{Introduction}

The differential expression of mucins has been related to altered risk of metastasis and death. Recently, mucins have been used as targets for molecular therapy and as a source of immune therapy [1]. The mucin family includes proteins that contain tandem repeat structures with a high proportion of prolines, threonines and serines. The human mucin (MUC) family consists of members-designated MUC1 to MUC21-that have been sub-classified into secreted and transmembrane forms. The secreted mucins (for example, MUC2, MUC5AC, MUC5B and MUC6) form a physical barrier, which as a mucous gel provides protection for epithelial cells that line the respiratory and gastrointestinal tracts and form the ductal surfaces of organs such as the liver, breast, pancreas and kidney. The transmembrane mucins (for example, MUC1, MUC4, MUC13 and MUC16) have a single membrane-spanning region and contribute to the protective mucous gel through their ectodomains of O-glycosylated tandem repeats that form rod-like structures [2]. Mucins would play a crucial role in forming the defense structure of the gut and could thus be well associated with its diseases. Inflammatory bowel disease [3], crohn's disease [4,5] and colorectal cancer [1,6] are known to have the involvement of mucins via different mechanisms. As much as it is established that mucins play a crucial role in causing colorectal cancer, the mechanism by which they do so is very complex. There are expression level studies that link mucins with colorectal cancer, but there are conflicting reports regarding their differential expression with respect to normal tissue.

There is evidence of upregulation of some mucins in breast, pancreatic and ovarian cancers especially the transmembrane ones. But there are reports about downregulation of mucins in colorectal cancer [7].

The posttranslational modification of mucins through glycosylation could be crucial in determining their role in cancer. Hence, most of the studies were intended to study the functional aspects of mucins and their association with cancer. This has also led to the identification of target mucins for cancer therapy.

The need for fully understanding the expression pattern of the mucin family genes is strongly felt especially in colorectal cancer where the mucin lined gut is the site of action. The present amount of evidence is not sufficient to establish the expression pattern of mucin family of genes in colorectal cancer at human level. In the present study, we attempt to fill this gap of knowledge by undertaking a comprehensive study focused at analyzing the mucins at transcript level. We studied the expression pattern of tumor tissues and compared it with the normal tissues derived from the same patients and found downregulation of all mucin family genes. This is the first report that comprehensively provides microarray based evidence for downregulation of mucins in colorectal cancer.

\section{Materials and Methods}

\section{Sample collection}

We collected 92 matched tumor-normal samples from 48 patients diagnosed with colorectal cancer. Each Hematoxylin and Eosin (H\&E) stained case was reviewed by a board-certified pathologist to confirm the specimen histological consistency with colon adenocarcinoma and that normal specimen contained no tumor cells. The sections were required to contain $>60 \%$ tumor cell nuclei for inclusion in the study. The study was granted the institutional review Board (IRB) approval after due review. The necessary procedural and ethical consent forms were signed by the patients prior to sample collection.

\section{Sample processing and DNA/RNA extraction}

Each specimen weighed between $10-30 \mathrm{mg}$. The tissue sample was stored in RNA later (Ambion) at $4^{\circ} \mathrm{C}$ for $24 \mathrm{hrs}$; followed by freezing and further storage at $-20^{\circ} \mathrm{C}$. DNA and RNA extraction was carried out from the same sample by NucleoSpin Trio Kit (Macherey-Nagel, Germany). Quality and quantity checks were done using Nanodrop (Thermo Fischer Scientific).

*Corresponding author: Mohammad Azhar Aziz, PO Box 22490, Mail Code 2216 King Abdul Aziz Medical City, Riyadh, 11426, Saudi Arabia, Tel: +966 18016030; Fax:+966 18011111 ext 16662; E-mail: azizmo@ngha.med.sa

Received May 26, 2014; Accepted September 21, 2014; Published September 27, 2014

Citation: Aziz MA, AlOtaibi M, AlAbdulrahman A, AlDrees M, AlAbdulkarim I (2014) Mucin Family Genes are Downregulated in Colorectal Cancer Patients. J Carcinogene Mutagene S10: 009. doi:10.4172/2157-2518.S10-009

Copyright: (C) 2014 Aziz MA, et al. This is an open-access article distributed under the terms of the Creative Commons Attribution License, which permits unrestricted use, distribution, and reproduction in any medium, provided the original author and source are credited. 
Citation: Aziz MA, AlOtaibi M, AlAbdulrahman A, AIDrees M, AlAbdulkarim I (2014) Mucin Family Genes are Downregulated in Colorectal Cancer Patients. J Carcinogene Mutagene S10: 009. doi:10.4172/2157-2518.S10-009

Page 2 of 7

\section{Data generation using human exon arrays}

For gene expression studies, Human Gene Chip Exon 1.0 ST arrays were obtained from Affymetrix. The recommended WT expression kit was obtained from Ambion. Recommended protocol for amplification, labeling and hybridization was followed. Arrays were scanned using $30007 \mathrm{G}$ scanner from Affymetrix. Data was exported using Expression Console in the form of .CEL files.

\section{Gene expression analysis}

'AltAnalyze' program (v2.8.0.1 -64bit) was used for exon level gene expression analysis. The exon expression intensities were summarized based on constitutive probe set expression to generate gene expression values. Finally, these values were utilized to calculate the differential expression of genes. Fold change values were calculated by creating a ratio using the signal intensities in tumor vs. normal samples.

\section{Integromics analysis}

Omics Office for biomarker discovery from TIBCO spotfire was used to carry out statistical analyses. Linear Models for Microarray Analysis (LIMMA) and Hierarchical clustering were carried out using Integromics.

\section{Ingenuity pathway analysis}

Data from AltAnalyze for expression levels of all mucin family genes was subjected to Ingenuity Pathway Analysis (IPA). Biomarker and Core analyses were carried out with a selection for 'Human' and 'colorectal cancer'.

\section{Results}

Mucin family genes are downregulated in colorectal cancer.

\begin{tabular}{|c|c|c|c|c|}
\hline Definition & Symbol & Fold-Change Tumor vs. Normal & Adj p-value Tumor vs. Normal & ANOVA \\
\hline mucin-like 1 & MUCL1 & 1.177928928 & 0.324880848 & 0.33269532 \\
\hline mucin 15 , cell surface associated & MUC15 & -1.008115774 & 0.928082382 & 0.927222838 \\
\hline mucin 16 , cell surface associated & MUC16 & -1.021494911 & 0.810097704 & 0.808270374 \\
\hline mucin 6 , oligomeric mucus/gel-forming & MUC6 & -1.059016589 & 0.272918223 & 0.258507815 \\
\hline mucin 19 , oligomeric & MUC19 & -1.088384059 & 0.287600842 & 0.286939379 \\
\hline mucin 7 , secreted & MUC7 & -1.088559983 & 0.455603832 & 0.459317975 \\
\hline mucin 8 & MUC8 & -1.090471283 & 0.070469366 & 0.062986147 \\
\hline mucin 21 , cell surface associated & MUC21 & -1.09606114 & 0.168972271 & 0.090273566 \\
\hline mucin 22 & MUC22 & -1.097767715 & 0.079416992 & 0.090273566 \\
\hline mucin 1 , cell surface associated & MUC1 & -1.139269043 & 0.168972271 & 0.172474892 \\
\hline mucin $3 \mathrm{~A}$, cell surface associated & MUC3A & -1.161091911 & 0.259114609 & 0.266051645 \\
\hline mucin 20 , cell surface associated & MUC20 & -1.240464797 & 0.207167498 & 0.215117389 \\
\hline mucin 13 , cell surface associated & MUC13 & -1.248437908 & 0.631733467 & 0.638485312 \\
\hline mucin 5B, oligomeric mucus/gel-forming & MUC5B & -1.743234796 & 0.005887836 & 0.007046705 \\
\hline mucin 12 , cell surface associated & MUC12 & -1.890092833 & 0.000218802 & 0.000293135 \\
\hline mucin 4 , cell surface associated & MUC4 & -2.209820943 & 0.000460973 & 0.000609726 \\
\hline mucin 2, oligomeric mucus/gel-forming & MUC2 & -3.531477506 & 0.000350047 & 0.000476223 \\
\hline
\end{tabular}

Table 1: All mucins except MUCL1 are downregulated in colorectal cancer patient samples.

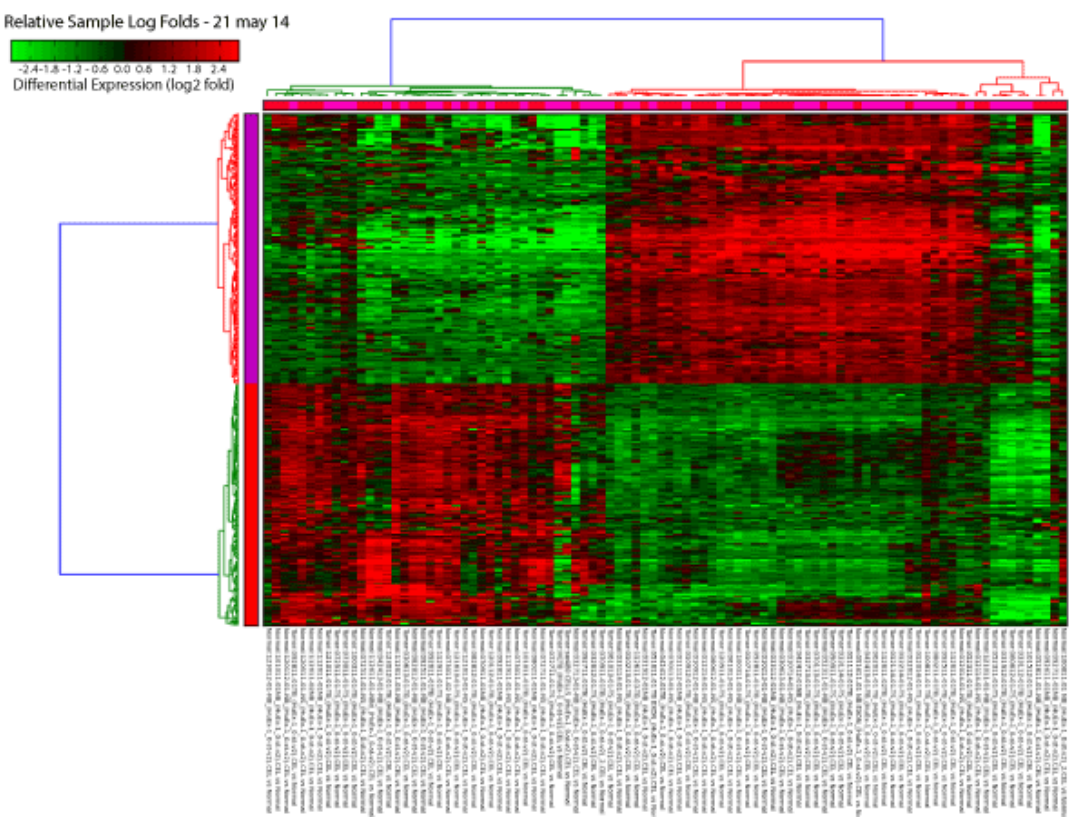

Figure 1: Clustering based on Relative sample log fold. All 92 samples were subjected to AltAnalyze software to generate this heatmap. The differential expression with a log fold range of -2.4 to 2.4 was used. Green colored area shows downregulated genes whereas Red shows upregulated genes. 
Citation: Aziz MA, AlOtaibi M, AlAbdulrahman A, AIDrees M, AlAbdulkarim I (2014) Mucin Family Genes are Downregulated in Colorectal Cancer Patients. J Carcinogene Mutagene S10: 009. doi:10.4172/2157-2518.S10-009

Page 3 of 7

\section{Analysis using AltAnalyze}

Analysis of 45,475 probe IDs available on exon array, made possible the composite analysis of mucin genes. 17 mucin genes were found in the database out which 16 were downregulated. Only MUCL1 was upregulated with a fold change value of 1.17 (Table 1).Differential expression heat map analysis shows two distinct groups from the 92 samples studied (Figure 1).

\section{Analysis using integromics}

Only Nine mucin genes were mapped by Integromics software. Hierarchical clustering analysis for these nine mucin genes using complete Euclidean linkage shows MUC20, MUC4, MUC1 and MUC13 with maximum scores (Figure 2).

LIMMA analysis was carried out to find out the most significant differentially expressed genes. Of all the nine mucin genes, MUC1 and MUC4 were found to be statistically most significant in terms of differential expression (Figure 3).

\section{Ingenuity pathway analysis}

In order to comprehensively analyze the functional significance of the downregulated mucin genes, we carried out ingenuity pathway analysis. Biomarker analysis for colorectal cancer in humans yielded MUC2 and MUC4 to be the putative genes (Table 2). They were found to be biomarkers for diagnosis, disease progression, efficacy and prognosis.

An upstream target analysis for mucin genes gave us a list of 35 genes, which could be affecting mucins through different pathways (Table 3). Top upstream regulators were CTCF, IL22, GAB2, IL1B and TSC2 genes.

Seven mucin genes viz., MUC1, MUC2, MUC3A, MUC4, MUC5B, MUC6 and MUC8 were part of a network with a score of 17 (The score is a numerical value used to rank networks according to their degree of relevance to the network eligible molecules, which are mucin genes mentioned above). Genes from this network could be involved in cellular development, cellular growth and proliferation, cell death and survival (Figure 4).

A network generated out of the mucin genes and overlaid with different cancer related functions showed involvement of MUC1, MUC2 and MUC4. However, MUC 3A, MUC 5B, MUC12, MUC19, MUC21 and MUCL1 were not associated with any known colorectal cancer related functions (Figure 5).

\section{Discussion}

Mucins are known to be involved in different types of cancer. There have been many reports about their role in causation or spread of cancer. However, there is no comprehensive attempt to measure the

\section{Hierarchical Cluster Analysis for Complete Linkage Euclidean for mucin genes}

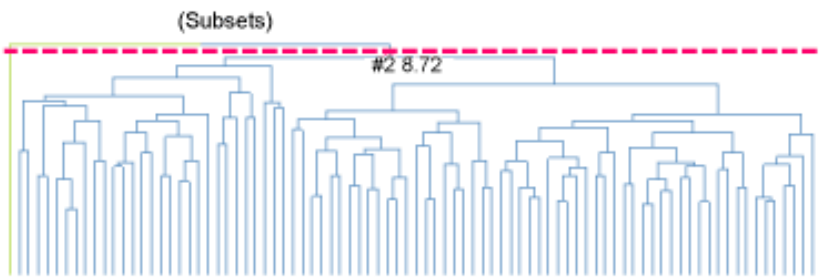

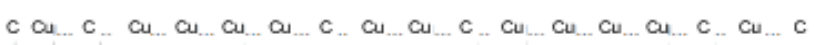
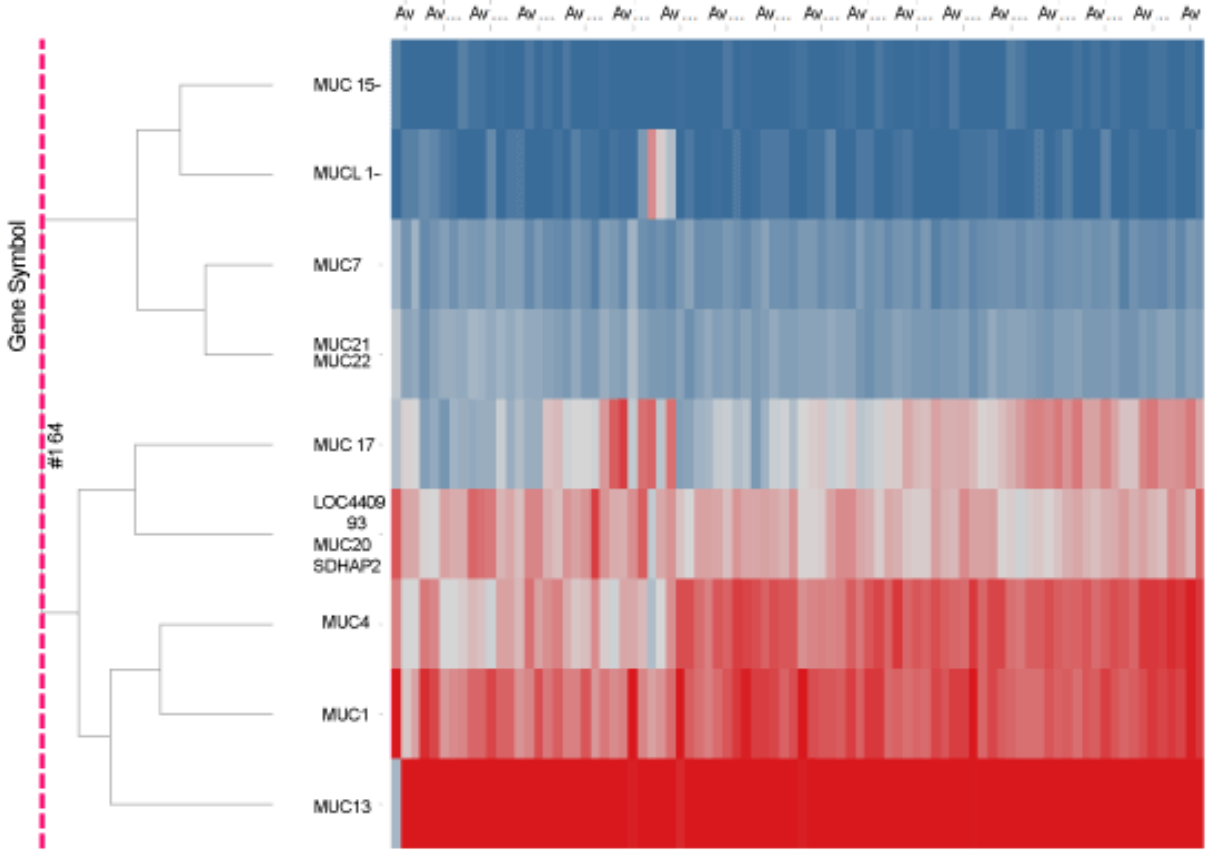

Figure 2: Hierarchical clustering analysis for mucin genes using complete Euclidean linkage. Using Integromics software all samples were clustered for mucin genes. MUC20, MUC4, MUC1 and MUC13 showed maximum score.

Data table: Clustering Complete Linkage Marking: Colors Max Average
Min

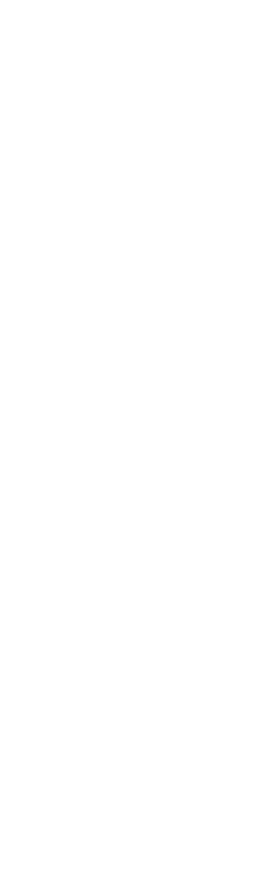




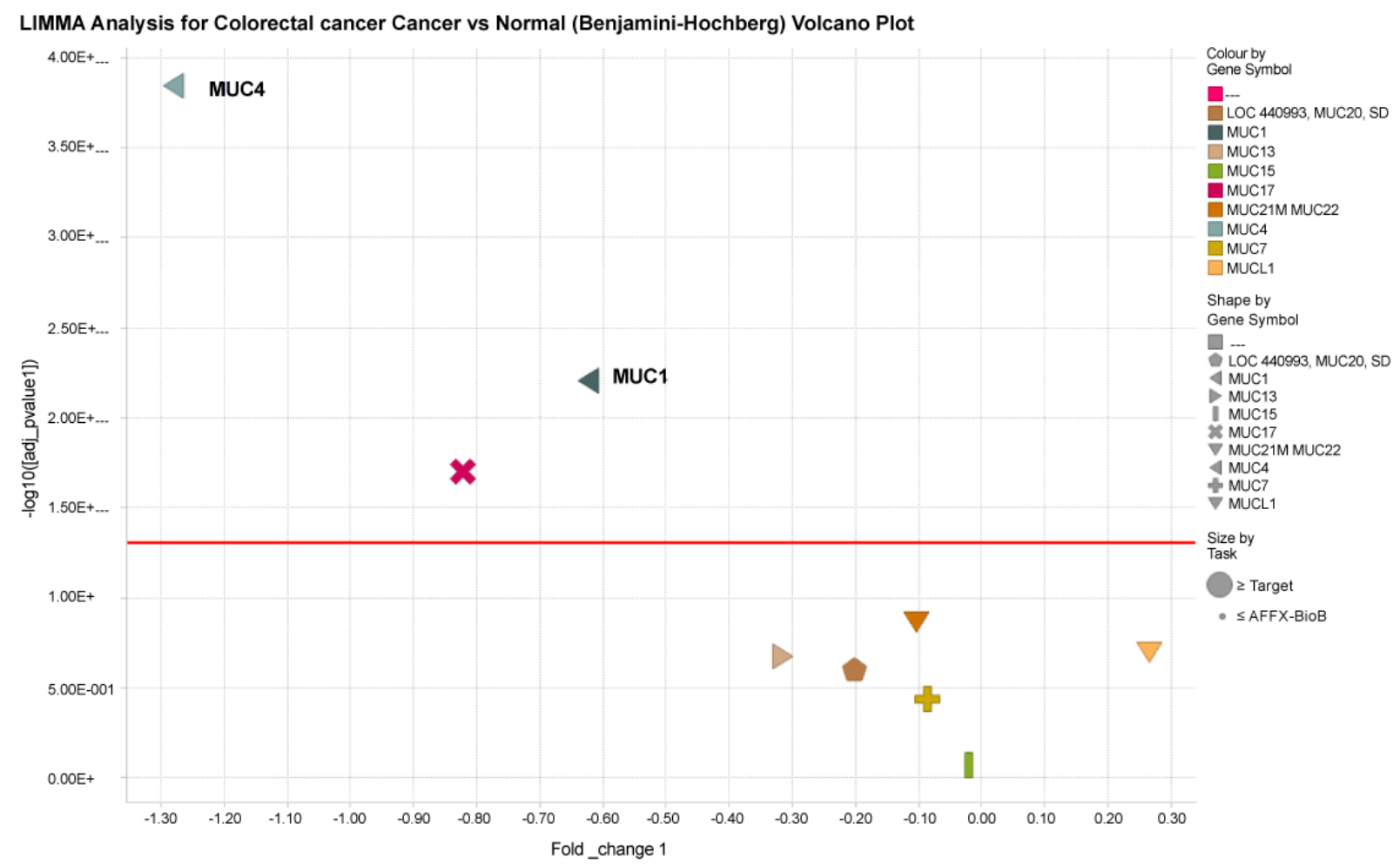

Figure 3: LIMMA analysis for mucin genes. Linear Models for Microarray analyses was done for mucin genes to analyse the statistical significance of differentially expressed genes. MUC4, MUC1 and MUC17 were found to be the most significant genes.

\begin{tabular}{|c|c|c|}
\hline Location & Plasma Membrane & Extracellular Space \\
\hline Family & transcription regulator & growth factor \\
\hline Drug(s) & HuHMFG1 & \\
\hline Fold Change & -1.139 & -2.210 \\
\hline p-value & $4.40 \mathrm{E}-02$ & $1.49 \mathrm{E}-05$ \\
\hline Human & $x$ & $x$ \\
\hline Blood & $x$ & \\
\hline BL Fluid & $x$ & \\
\hline Plasma/Serum & $x$ & \\
\hline Sputum & $x$ & \\
\hline Tears & & $x$ \\
\hline Urine & $x$ & \\
\hline Memory B cells & $x$ & \\
\hline Naive B cells & $x$ & \\
\hline Epidermis & $x$ & \\
\hline Kidney & $x$ & \\
\hline Large Intestine & & $x$ \\
\hline Lung & $x$ & \\
\hline Lymph node & $x$ & \\
\hline Mammary Gland & $x$ & \\
\hline Ovary & $x$ & \\
\hline Pancreas & $x$ & \\
\hline Retina & & $x$ \\
\hline Skin & $x$ & \\
\hline Small Intestine & $x$ & \\
\hline Stomach & $x$ & \\
\hline Thyroid Gland & & $x$ \\
\hline Uterus & & $x$ \\
\hline COLO205 & $x$ & \\
\hline HCC-2998 & $x$ & \\
\hline HT29 & $x$ & \\
\hline $\mathrm{KM}-12$ & $x$ & \\
\hline SW-620 & $x$ & \\
\hline
\end{tabular}

Table 2: Putative biomarker molecules among the family of mucin genes.
RNA expression level analysis for the entire mucin family genes in the same setting. In this report we have attempted to study the expression level of all mucin family genes in colorectal cancer patient samples. We employed the exon microarray to interrogate the expression levels in 92 samples. AltAnalyze and Integromics softwares produced consistent results providing a confident analysis of mucin family genes.

MUC1 has been implicated in colorectal and other cancers mainly by studies involving post transcriptional events. Some studies suggest the gene expression changes of its variants in cancer as well. MUC1 is being found to be an important molecule in the immunobiology of adenocarcinomas [8]. The role of MUC1 in cancer progression is still controversial and somewhat confusing. While Mukherjee and colleagues developed MUC1 specific immune therapy in a CRC model, Lillehoj and co-investigators showed recently that MUC1 inhibits cell proliferation by a $\beta$-catenin-dependent mechanism [9]. MUC1 has been suggested as a potential target for developing anticancer agents including vaccines, antibodies and small molecules [10,11]. Several lines of evidence point towards a biological role of MUC1 in colorectal cancer. A positive correlation was described between mucin secretion, proliferation, invasiveness, metastasis and bad prognosis [12-14]. In another report, mucinous CRC is associated with a higher expression of MUC2 and MUC5AC, but a lower expression of MUC1 [1]. Mucin 1 (Muc1) is a tumor-associated glycoprotein and has an important role in cancer progression and metastasis. Recent evidence suggests inhibition of Mucl could significantly suppress the growth of HCC2998 in nude mice models [15]. Our results show MUC1 to be downregulated in tumor samples, which is consistent with some studies but not all.

Studies at protein level show overexpression of MUC2, MUC5B, MUCAC and MUC6 in colorectal cancer [16]. Immunohistochemical 
Citation: Aziz MA, AlOtaibi M, AlAbdulrahman A, AIDrees M, AlAbdulkarim I (2014) Mucin Family Genes are Downregulated in Colorectal Cancer Patients. J Carcinogene Mutagene S10: 009. doi:10.4172/2157-2518.S10-009

Page 5 of 7

\begin{tabular}{|c|c|c|c|}
\hline Upstream Regulator & Molecule Type & p-value of overlap & Target molecules in dataset \\
\hline CTCF & transcription regulator & $1.43 \mathrm{E}-08$ & MUC2,MUC5B,MUC6 \\
\hline IL22 & cytokine & $4.90 \mathrm{E}-08$ & MUC1,MUC4,MUC5B \\
\hline GAB2 & other & 1.77E-06 & MUC2,MUC5B \\
\hline IL1B & cytokine & 2.06E-04 & MUC1,MUC3A,MUC4 \\
\hline TSC2 & other & 7.93E-04 & MUC2 \\
\hline FOXA1 & transcription regulator & 7.93E-04 & MUC4 \\
\hline AGR2 & other & $1.59 \mathrm{E}-03$ & MUC1 \\
\hline DDIT4 & other & $1.59 \mathrm{E}-03$ & MUC2 \\
\hline CDX1 & transcription regulator & $1.59 \mathrm{E}-03$ & MUC4 \\
\hline SP1 & transcription regulator & $2.58 \mathrm{E}-03$ & MUC2,MUC5B \\
\hline NFATC3 & transcription regulator & 3.17E-03 & MUC2 \\
\hline RPS6KB1 & kinase & 3.17E-03 & MUC1 \\
\hline Secretase gamma & complex & 3.96E-03 & MUC2 \\
\hline DNMT3A & enzyme & $3.96 \mathrm{E}-03$ & MUC4 \\
\hline HNF1B & transcription regulator & 3.96E-03 & MUC4 \\
\hline SIM2 & transcription regulator & 4.75E-03 & MUC2 \\
\hline ATF1 & transcription regulator & $6.33 \mathrm{E}-03$ & MUC5B \\
\hline $\begin{array}{c}\text { miR-145-5p (and other miRNAs w/seed } \\
\text { UCCAGUU) }\end{array}$ & mature microRNA & $7.12 \mathrm{E}-03$ & MUC1 \\
\hline ERBB2 & kinase & 7.12E-03 & MUC1 \\
\hline IL13 & cytokine & 8.66E-03 & MUC2,MUC5B \\
\hline MTOR & kinase & $9.48 \mathrm{E}-03$ & MUC1 \\
\hline HDAC3 & transcription regulator & $9.48 \mathrm{E}-03$ & MUC4 \\
\hline PRKCD & kinase & $1.26 \mathrm{E}-02$ & MUC5B \\
\hline HNF4A & transcription regulator & $1.26 \mathrm{E}-02$ & MUC4 \\
\hline Akt & group & $1.50 \mathrm{E}-02$ & MUC1 \\
\hline CAMP & other & $1.81 \mathrm{E}-02$ & MUC2 \\
\hline $\mathrm{CDX} 2$ & transcription regulator & $2.82 \mathrm{E}-02$ & MUC4 \\
\hline GATA4 & transcription regulator & $2.90 \mathrm{E}-02$ & MUC4 \\
\hline HDAC1 & transcription regulator & $2.90 \mathrm{E}-02$ & MUC4 \\
\hline SP3 & transcription regulator & $3.28 \mathrm{E}-02$ & MUC2 \\
\hline HIF1A & transcription regulator & 3.67E-02 & MUC1 \\
\hline TLR2 & transmembrane receptor & $3.89 E-02$ & MUC2 \\
\hline ERK & group & $4.28 \mathrm{E}-02$ & MUC8 \\
\hline DNMT3B & enzyme & 4.35E-02 & MUC4 \\
\hline Histone h3 & group & 4.73E-02 & MUC4 \\
\hline
\end{tabular}

Table 3: Upstream regulators for mucin genes.

studies have been attempted to use the expression pattern for diagnostic value. However, it came with a heterogeneous pattern for MUC1, MUC2 and MUC25AC [17]. Studies with knockout mice suggest the tumor suppressing nature for MUC2 [18] and is supported by other studies [19]. This also resonates in our results which show downregulation of MUC2 in tumor samples. Using in situ hybridization and immunohistochemistry, it was shown that MUC2 and MUC3 were downregulated in colorectal cancer [20]. MUC 11 and 12 were also found downregulated in colorectal cancer [7]. Immunohistochemical patterns have also shown that MUC4 was downregulated in hyperplastic polyps and completely lost in serrated adenomas [21].

MUC12 gene expression was also earlier reported to be downregulated in colorectal cancers and its probable association with metastasis [22]. MUC12 was also found to be an independent marker for prognosis in stage II and III colorectal cancer patients. We also report the downregulation of MUC12 in a significant manner.

The current level of evidence linking mucins to colorectal cancer underlies the importance of studying these molecules in detail. However, the disparity in evidences regarding the expression and post translational modification of these mucins in colorectal cancer may be limiting factor. These disparities could be because of difference in sampling and the method of investigation. This generates a big scope to study these mucins in a composite manner. Our study attempts to address this using a single snapshot taken from tumor and normal samples using exon level microarray. Ingenuity pathway analysis put these mucins in a different perspective with spotlight on MUC1 and MUC4 for their use as biomarkers. There are still some unexplored mucins with highly significant differential expression (e.g. MUC5B) that deserve further studies to prove or rule out their importance in colorectal cancer. 
Citation: Aziz MA, AlOtaibi M, AlAbdulrahman A, AIDrees M, AlAbdulkarim I (2014) Mucin Family Genes are Downregulated in Colorectal Cancer Patients. J Carcinogene Mutagene S10: 009. doi:10.4172/2157-2518.S10-009

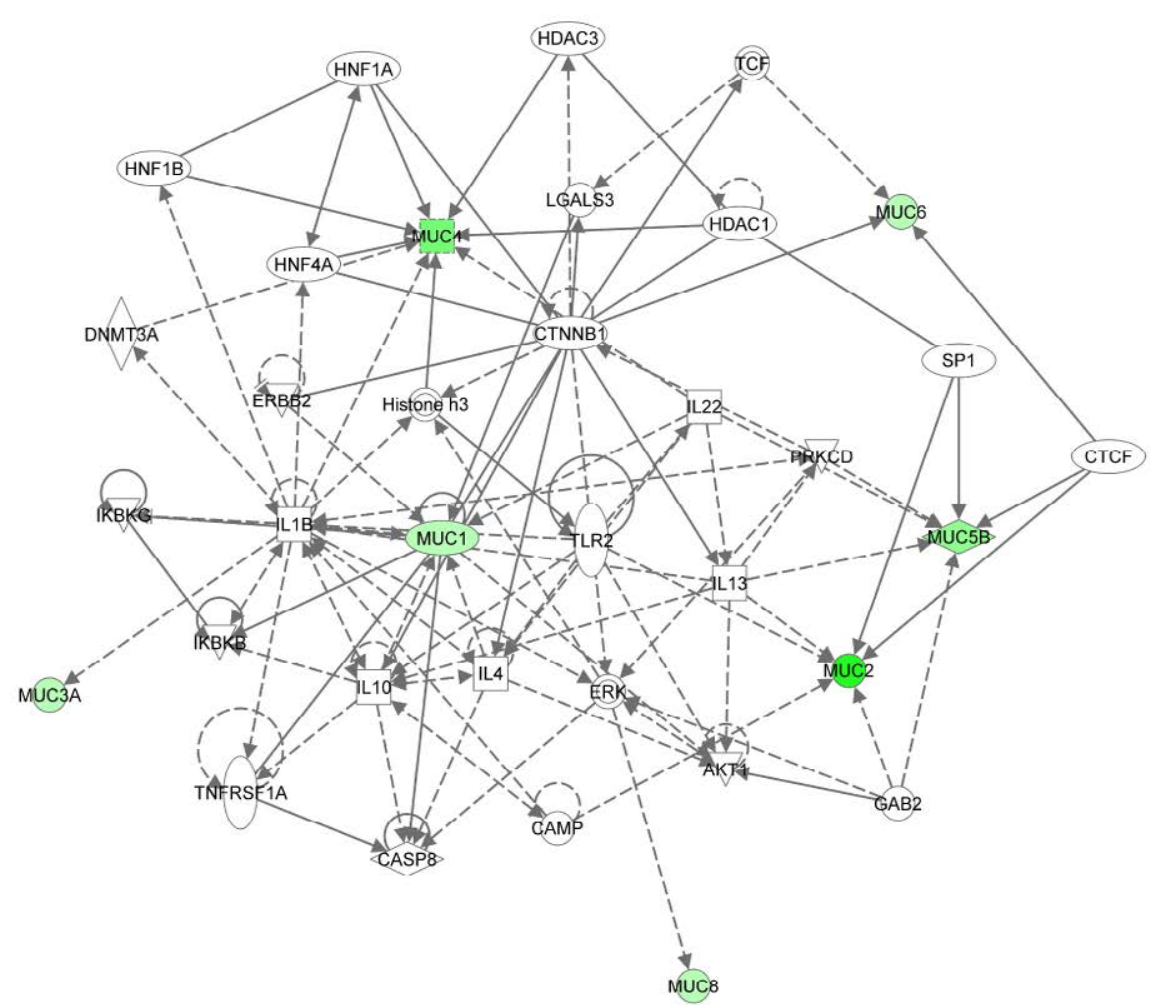

Figure 4: Network analysis of mucin genes. Using Ingenuity Pathway analysis all mucin genes were subjected to core analysis. $7 / 17$ genes were included in the network with other molecules.

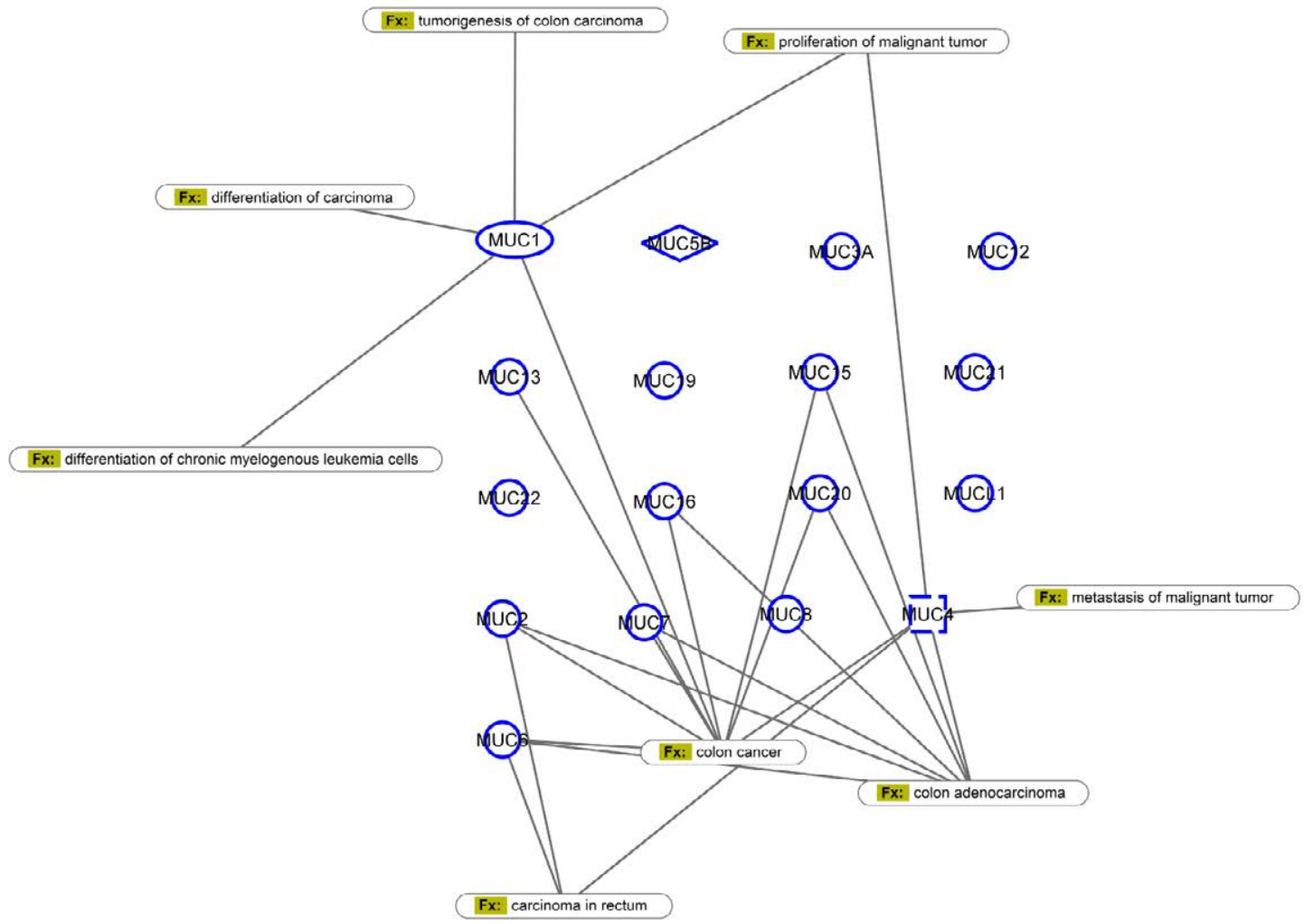

Figure 5: Mucin genes associated with colorectal cancer related functions. Mucin genes were overlaid with known functions associated with different aspects of colorectal cancer. Some of these mucin genes show little or no known role in colorectal cancer. Genes with high significance and unknown role would be the molecules with priority to be studied further. 
Citation: Aziz MA, AlOtaibi M, AlAbdulrahman A, AIDrees M, AlAbdulkarim I (2014) Mucin Family Genes are Downregulated in Colorectal Cancer Patients. J Carcinogene Mutagene S10: 009. doi:10.4172/2157-2518.S10-009

\section{Acknowledgement}

This study was funded by King Abdullah Intl Med Res Ctr through grant\#RC10/083 awarded to MAA. We thank Drs. Abdulkarim and Balwi for their help in providing necessary infrastructure. None of the authors have any conflict of interest.

\section{References}

1. Debunne H, Ceelen W (2013) Mucinous differentiation in colorectal cancer: molecular, histological and clinical aspects. Acta Chir Belg 113: 385-390.

2. Kufe DW (2009) Mucins in cancer: function, prognosis and therapy. Nat Rev Cancer 9: 874-885.

3. Sheng YH, Hasnain SZ, Florin TH, McGuckin MA (2012) Mucins in inflammatory bowel diseases and colorectal cancer. J Gastroenterol Hepatol 27: 28-38.

4. Buisine MP, Desreumaux P, Leteurtre E, Copin MC, Colombel JF, et al. (2001) Mucin gene expression in intestinal epithelial cells in Crohn's disease. Gut 49: 544-551.

5. Buisine MP, Desreumaux P, Debailleul V, Gambiez L, Geboes K, et al. (1999) Abnormalities in mucin gene expression in Crohn's disease. Inflamm Bowe Dis 5: 24-32.

6. Kawashima H (2012) Roles of the gel-forming MUC2 mucin and its O-glycosylation in the protection against colitis and colorectal cancer. Bio Pharm Bull 35: 1637-1641.

7. Williams SJ, McGuckin MA, Gotley DC, Eyre HJ, Sutherland GR, et al. (1999) Two novel mucin genes down-regulated in colorectal cancer identified by differential display. Cancer Res 59: 4083-4089.

8. Finn OJ, Gantt KR, Lepisto AJ, Pejawar-Gaddy S, Xue J, et al. (2011) Importance of MUC and spontaneous mouse tumor models for understanding the immunobiology of human adenocarcinomas. Immunol Res 50: 261-268.

9. Niv Y (2008) MUC1 and colorectal cancer pathophysiology considerations. World J Gastroenterol 14: 2139-2141.

10. Kufe DW (2009) Functional targeting of the MUC1 oncogene in human cancers Cancer Biol Ther 8: 1197-1203.

11. Beatty P, Ranganathan S, Finn OJ (2012) Prevention of colitis-associated colon cancer using a vaccine to target abnormal expression of the MUC1 tumor antigen. Oncoimmunology 1: 263-270.
12. Niv Y, Schwartz B, Amsalem Y, Lamprecht SA (1995) Human HT-29 colon carcinoma cells: mucin production and tumorigenicity in relation to growth phases. Anticancer Res 15: 2023-2027.

13. Duncan TJ, Watson NF, Al-Attar AH, Scholefield JH, Durrant LG (2007) Th role of MUC1 and MUC3 in the biology and prognosis of colorectal cancer. World J Surg Oncol 5: 31.

14. Bresalier RS, Niv Y, Byrd JC, Duh QY, Toribara NW, et al. (1991) Mucin production by human colonic carcinoma cells correlates with their metastatic potential in animal models of colon cancer metastasis. J Clin Invest 87:10371045

15. Wang Z, Sun J, Hu X, Huang S (2014) Interference of mucin 1 inhibits progression of colon carcinoma by repression of $\mathrm{Wnt} / \mathrm{\beta}$-catenin signaling. DNA Cell Biol 33: 162-170.

16. Walsh MD, Clendenning M, Williamson E, Pearson SA, Walters RJ, et al. (2013) Expression of MUC2, MUC5AC, MUC5B, and MUC6 mucins in colorecta cancers and their association with the $\mathrm{CpG}$ island methylator phenotype. Mod Pathol 26: 1642-1656.

17. Lau SK, Weiss LM, Chu PG (2004) Differential expression of MUC1, MUC2, and MUC5AC in carcinomas of various sites: an immunohistochemical study. Am J Clin Pathol 122: 61-69.

18. Velcich A, Yang W, Heyer J, Fragale A, Nicholas C, et al (2002) Colorecta cancer in mice genetically deficient in the mucin Muc2. Science 295: 17261729.

19. Byrd JC, Bresalier RS (2004) Mucins and mucin binding proteins in colorectal cancer. Cancer Metastasis Rev 23: 77-99.

20. Chang SK, Dohrman AF, Basbaum CB, Ho SB, Tsuda T, et al. (1994) Localization of mucin (MUC2 and MUC3) messenger RNA and peptide expression in human normal intestine and colon cancer. Gastroenterology 107 28-36.

21. Biemer-Huttmann AE, Walsh MD, McGuckin MA, Ajioka $Y$, Watanabe $\mathrm{H}$, et a (1999). Immunohistochemical staining patterns of MUC1, MUC2, MUC4, and MUC5AC mucins in hyperplastic polyps, serrated adenomas, and traditional adenomas of the colorectum. J Histochem Cytochem 47:1039-1048.

22. Matsuyama T, Ishikawa T, Mogushi K, Yoshida T, lida S, et al. (2010) MUC12 mRNA expression is an independent marker of prognosis in stage II and stage III colorectal cancer. Int J Cancer 127: 2292-2299. 